\title{
Medical Treatments for Idiopathic Pulmonary Fibrosis: a Protocol for a Systematic Review and Network Meta-analysis
}

\section{Tyler Pitre ( $\square$ tyler.pitre@medportal.ca )}

McMaster University https://orcid.org/0000-0003-3015-0723

Jasmine Mah

Dalhousie Medical School: Dalhousie University Faculty of Medicine

Sonya Cui

McMaster University Michael G DeGroote School of Medicine

Melanie C. Zhang

McMaster University Michael G DeGroote School of Medicine

\section{Renata Husnudinov}

McMaster University Michael G DeGroote School of Medicine

\section{Faran Khalid}

McMaster University Michael G DeGroote School of Medicine

\section{Wryan Helmeczi}

uOttawa Faculty of Medicine: University of Ottawa Faculty of Medicine

Johnny Su

McMaster University Department of Medicine

\section{Laura Banfield}

McMaster University

\section{Aaron Jones}

McMaster University Department of Health Research Methods Evidence and Impact

\section{Protocol}

Keywords: IPF, medical therapies, mortality, FVC, FEV1

Posted Date: April 20th, 2021

DOl: https://doi.org/10.21203/rs.3.rs-420792/v1

License: (c) (1) This work is licensed under a Creative Commons Attribution 4.0 International License.

Read Full License 


\section{Abstract}

Background: Idiopathic pulmonary fibrosis (IPF) is a respiratory disorder of unknown etiology with a poor prognosis. There are novel therapies that have been studied in randomized controlled trials since the last network meta-analysis that may be of interest to academics and clinicians.

Methods: We will perform a network meta-analysis on eligible randomized controlled trials of patients with IPF. We intend to search MEDLINE, EMBASE, Cochrane and clinicaltrials.org in order to complete a comprehensive search for adult IPF patients being treated with at least one of 21 of the selected medical therapies. A team will screen and extract eligible trials. We will perform Bayesian random-effects network meta-analysis. We will use GRADE and RoB 2.0 to assess the certainty and quality of the evidence.

Discussion: There is a need for an updated meta-analysis on IPF medical therapies, including novel medical therapies. We intend on studying up to 21 medical therapies in the network meta-analysis to provide the most accurate and updated summary of the evidence for IPF treatments.

\section{Systematic review registrations: https://osf.io/afbhd/}

\section{Background:}

Idiopathic pulmonary fibrosis (IPF) is a progressive interstitial pneumonia of unknown etiology with a poor prognosis, despite the introduction of novel antifibrotic medications $(1,2)$. The clinical manifestations of IPF include nonspecific respiratory symptoms such as worsening dyspnea and cough $(1,2)$. Overtime, the disease is marked by a progressive decline in pulmonary function, with increasing acute exacerbations, leading eventually to death. With the introduction of pirfenidone and nintedanib, there has been improvement in IPF care; otherwise there have been a myriad of proposed therapies which have had mixed results in the literature. In addition, over the past few years, there has also been several novel and understudied therapies as well with unclear benefit $(3,4,5)$.

There have been a number of network meta-analyses on IPF therapies. One included 10 treatments for IPF, including nintedanib and pirfenidone, but also a variety of others, and found that treatment with nintedanib, pirfenidone, and sildenafil extends survival in patients with IPF (6). Another NMA looking at 16 drug therapies suggested that only pirfenidone and nintedanib had a trend towards decreasing overall mortality (7). Interestingly, many of the network meta-analyses have demonstrated discordant results in terms of beneficial therapies. Since then, additional treatment options have surfaced but they have not been formally studied in a large network meta-analysis $(3,4,5,8,9,11)$.

We aim to perform a multiple drug treatment comparison based on a network meta-analysis considering both direct and indirect comparisons of 21 treatment interventions that have been tested in randomized controlled trials of adult patients with well-defined IPF. We focused our outcomes based on mortality, markers of disease severity and adverse effects leading to treatment discontinuation. 


\section{Methods:}

This protocol is registered in Open Science Framework (OSF) at https://osf.io/afbhd/. For the development of the present protocol, we used the Preferred Reporting Items for Systematic Review and Meta-Analysis Protocols (PRISMA-P).

\section{Objective}

To evaluate and estimate relative comparisons of the direct and indirect evidence supporting 21 approved and studied treatments for IPF for the outcomes of mortality, disease progression and adverse events.

\section{Search strategy}

A systematic literature review of primary studies was conducted up to March 2021. The databases selected were relevant to the review topic and types of eligible studies. The electronic search was first developed in the bibliographic database MEDLINE in consultation with a professional librarian. The search was translated into EMBASE, Cochrane Library Central/SR, and Clinicaltrials.gov.

A search strategy using both key text words and indexed standardized subject terms was used. In consultation with experts in IPF, the key concepts of IPF and relevant treatments were identified. Specifically, the definition of diagnosed adult IPF used in this review is defined by either the 2011 or 2018 ATS diagnostic criteria (11). A broad set of search terms were gathered for the first two concepts including generic and trade names for the proposed treatments and then cross referenced with existing systematic reviews on similar topics (3). Finally, a validated randomized control trial search filter was applied. Unpublished data was not included in our search strategy, although each full text paper included had a hand searched bibliography for additional papers.

\section{Eligibility criteria}

Bibliographic records screened were eligible for inclusion if the population included in the study were adults $>=18$ years of age diagnosed with IPF with past and present ATS diagnostic criteria, and the intervention had to include one or more of the following treatments listed in Appendix 1. These were chosen based on available clinical trials, expert opinion, and gaps in the literature.

The comparison group is placebo or standard care. Outcomes were not used to determine eligibility criteria to avoid bias from selection criteria. However, it was pre-determined that "critical outcomes" were mortality rates, disease progression (as measured by pulmonary function tests, functional ability/walk test, hospitalizations, or exacerbations) and adverse events from the treatments. The studies included were phase 2 or 3 randomized control trials (RCTs). This was chosen to provide the highest quality evidence for a research question of this nature, RCTS are the best way to minimize bias and confounders and are currently the gold standard for evaluating the efficacy of interventions.

\section{Study records:}




\section{Data management \& selection process}

References were uploaded to COVIDENCE, a systematic review web platform, where duplicate records were removed. Two independent authors used COVIDENCE to screen titles and abstracts independently for inclusion based on the PICOS design above. After screening, full texts were obtained and assessed for eligibility after being uploaded to COVIDENCE. Discrepancies in screening at both stages were resolved through consensus mediated by a third party.

\section{Data collection process \& data outcomes}

The following data will be extracted by the author using a modified version of Cochrane's template data collection form for intervention reviews of RCTs (Cochrane Collaboration, 2020). The initial form was piloted using a sample of the included primary studies by authors TP and JM. We did not reach out to authors for more information. (Appendix 2)

\section{Outcomes and prioritization}

The primary outcomes included all-cause mortality, reduction in disease progression, and serious adverse effects (SAE). Disease progression was chosen as a mean change in forced vital capacity (FVC), forced expiratory time at 1 second (FEV1), 6-minute walk test (6MWT), diffusion capacity of carbon monoxide (DLCO), number of hospitalizations and exacerbations. We will select outcomes closest to 24 weeks follow up.

\section{Risk of bias in individual studies}

For each included trial, reviewers will use a revision of the Cochrane tool for assessing risk of bias in randomized trials (RoB 2.0) to give a rating to trials (11). These ratings will be as either at i) low risk of bias, ii) some concerns (probably low risk of bias) iii) some concerns (probably high risk of bias) or iv) high risk of bias, across the following domains: bias arising from the randomization process; bias owing to departures from the intended intervention; bias from missing outcome data; bias in measurement of the outcome; bias in selection of the reported results, including deviations from the registered protocol; bias due to competing risks; and bias arising from early termination for benefit. Reviewers will resolve discrepancies by discussion and, when not possible, with adjudication by a third party.

\section{Data synthesis}

We will perform Bayesian random-effects network meta-analysis using the R package gemtc, version 0.82.(12). We will summarize the effect of interventions on our dichotomous outcome (i.e. mortality) using pooled odds ratio (OR) and corresponding $95 \%$ credibility intervals. For our continuous outcome, we will use the pooled mean differences (MD) and corresponding $95 \%$ credibility intervals. Treatment nodes will be grouped based on class of medication (12). If combination treatments were used, we will include that as a separate node. 
We will assess convergence using trace plots and will examine publication bias using funnel plots and the Ediger bias test. While we intend to utilize a random-effects model, we may opt for a fixed-effects model if the random effects analysis produces implausible credibility intervals due to the sparseness of the networks. We plan to perform meta-regressions as appropriate and subgroup analyses based on patient comorbidities as well.

\section{Confidence in cumulative evidence}

We will assess the certainty of the evidence with the grading of recommendations assessment, development and evaluation (GRADE) approach for network meta-analysis (13). The GRADE approach involves separate grading of quality of evidence for each patient-important outcome followed by determining an overall quality of evidence across outcomes. We plan to have two people rate each domain for each comparison separately and resolve discrepancies by consensus. We plan to rate the certainty for each comparison and outcome as high, moderate, low, or very low, based on considerations of risk of bias, inconsistency, indirectness, publication bias, intransitivity, incoherence (difference between direct and indirect effects), and imprecision.

\section{Discussion:}

In the last 5 years, there has been informative research in evaluating the clinical efficacy of IPF therapies. In 2016, there were two published NMAs evaluating the evidence of up to 16 different drug therapies (3, 4). One NMA concluded that nintedanib, pirfenidone, and sildenafil extends survival in patients with IPF while the other suggested only pirfenidone and nintedanib were approaching statistical significance when evaluating all-cause mortality $(6,7)$. In addition, there have been numerous studies suggesting the relative efficacy of both pirfenidone and nintedanib in treating IPF (14).

However, there have been numerous new therapies studied in the treatment of IPF since the last review and there are on-going clinical trials with novel therapies. The results of some of the novel therapies come with mixed results and unclear clinical benefit in terms of overall survival, disease progression and adverse outcomes $(4,5,8,9,10)$.

Our review provides an update of the previous reviews by including the previously studied therapies as well as 10 additional therapies that have been studied since then. Our review will report on mortality data, as well as markers of disease progression and adverse event data.

Our target users are clinicians with IPF patients, making evidence-based decisions on IPF therapies and to researchers on which novel drug therapies demonstrate promise in the burgeoning medical literature of IPF drug therapies.

Limitations of our study include a diverse patient population, with varying underlying comorbidities, as well as varying disease severity of the participants of each individual RCT. In addition, our study does not address many novel therapies and on-going trials of drug therapies with potential benefit. 


\section{Declarations:}

Ethics approval and consent to participate: Not applicable.

Consent for publication: Not applicable

Availability of data and material: Upon request to the corresponding author

Competing interests: None

Funding: None

Authors' contributions: TP is the main author. He came up with the study design, developed the methods, recruited the team and led the analytics. He helped write, edit and approve the protocol. JM helped design the methods, she developed the search strategy in conjunction with a professional librarian and trained the data collectors on risk of bias assessment. She helped write, edit and approve the protocol. SC, MZ, $\mathrm{RH}, \mathrm{MK}, \mathrm{WH}$, and JS screened abstracts, full text screening and performed data collection. They also performed the risk of bias assessment. All of these tasks were adjudicated and reviewed by TP and JM. LB provided expertise in search strategy and data collection. AJ is the analytic lead, performing data analysis, and consulting on methodology.

Acknowledgements: I would like to acknowledge Dena Zeraatkar for her critical and invaluable feedback.

\section{References:}

1. Richeldi L, Collard HR, Jones MG. Idiopathic pulmonary fibrosis. Lancet. 2017 May 13;389(10082):1941-52.

2. King TE, Pardo A, Selman M. Idiopathic pulmonary fibrosis. Lancet. 2011 Dec 3;378(9807):1949-61.

3. Somogyi V, Chaudhuri N, Torrisi SE, Kahn N, Müller V, Kreuter M. The therapy of idiopathic pulmonary fibrosis: what is next? Eur Respir Rev. 2019 Sep 30;28(153).

4. Raghu G, van den Blink B, Hamblin MJ, Brown AW, Golden JA, Ho LA, et al. Effect of recombinant human pentraxin 2 vs placebo on change in forced vital capacity in patients with idiopathic pulmonary fibrosis: a randomized clinical trial. JAMA. 2018 Jun 12;319(22):2299-307.

5. Raghu G, Scholand MB, de Andrade J, Lancaster L, Mageto Y, Goldin J, et al. FG-3019 anti-connective tissue growth factor monoclonal antibody: results of an open-label clinical trial in idiopathic pulmonary fibrosis. Eur Respir J. 2016 May;47(5):1481-91.

6. Rochwerg B, Neupane B, Zhang Y, Garcia CC, Raghu G, Richeldi L, et al. Treatment of idiopathic pulmonary fibrosis: a network meta-analysis. BMC Med. 2016 Feb 3;14:18.

7. Canestaro WJ, Forrester SH, Raghu G, Ho L, Devine BE. Drug treatment of idiopathic pulmonary fibrosis: systematic review and network meta-analysis. Chest. 2016 Mar;149(3):756-66. 
8. Raghu G, Brown KK, Collard HR, Cottin V, Gibson KF, Kaner RJ, et al. Efficacy of simtuzumab versus placebo in patients with idiopathic pulmonary fibrosis: a randomised, double-blind, controlled, phase 2 trial. Lancet Respir Med. 2017 Jan;5(1):22-32.

9. Raghu G, Brown KK, Costabel U, Cottin V, du Bois RM, Lasky JA, et al. Treatment of idiopathic pulmonary fibrosis with etanercept: an exploratory, placebo-controlled trial. Am J Respir Crit Care Med. 2008 Nov 1;178(9):948-55.

10. Maher TM, van der Aar EM, Van de Steen O, Allamassey L, Desrivot J, Dupont S, et al. Safety, tolerability, pharmacokinetics, and pharmacodynamics of GLPG1690, a novel autotaxin inhibitor, to treat idiopathic pulmonary fibrosis (FLORA): a phase 2a randomised placebo-controlled trial. Lancet Respir Med. 2018 Aug;6(8):627-35.

11. Sterne JA, Savović J, Page MJ, Elbers RG, Blencowe NS, Boutron I, et al. RoB 2: a revised tool for assessing risk of bias in randomised trials. BMJ. 2019 Aug 28;366:14898.

12. Efthimiou O, Debray TP, Valkenhoef G van, Trelle S, Panayidou K, Moons KG, et al. GetReal in network meta-analysis: a review of the methodology. Research Synthesis Methods. 2016;7(3):236-63.

13. Brignardello-Petersen R, Bonner A, Alexander PE, Siemieniuk RA, Furukawa TA, Rochwerg B, et al. Advances in the GRADE approach to rate the certainty in estimates from a network meta-analysis. Journal of Clinical Epidemiology. 2018 Jan 1;93:36-44.

14. Rogliani P, Calzetta L, Cavalli F, Matera MG, Cazzola M. Pirfenidone, nintedanib and N-acetylcysteine for the treatment of idiopathic pulmonary fibrosis: a systematic review and meta-analysis. Pulm Pharmacol Ther. 2016 Oct;40:95-103.

\section{Appendix:}

Appendix 1 is not available with this version.

\section{Supplementary Files}

This is a list of supplementary files associated with this preprint. Click to download.

- Appendix2.docx

- PRISMAP2015checklist.docx 